\title{
REDUCED INFECTION RATE AFTER ASEPTIC REVISION TOTAL HIP ARTHROPLASTY WITH EXTENDED ORAL ANTIBIOTIC PROTOCOL
}

\author{
Amer Mohiuddin $\mathrm{BS}^{1}$, Justin Rice $\mathrm{BA}^{1}$, \\ Mary Ziemba-Davis $\mathrm{BA}^{2}$, R. Michael Meneghini $\mathrm{MD}^{1,2}$ \\ ${ }^{1}$ Indiana University School of Medicine, Indianapolis, IN \\ ${ }^{2}$ IU Health Physicians, Orthopedics \& Sports Medicine, Fishers, IN
}

\begin{abstract}
Background and Hypothesis: Periprosthetic joint infection (PJI is a leading cause of failure after aseptic revision total hip arthroplasty (RTHA). While well documented in the primary setting, perioperative antibiotic duration is not well described in RTHA where the risk of PJI was recently reported to be $8 \%$ oneyear post-revision. The study purpose was to evaluate whether extended oral antibiotic prophylactic protocol minimizes PJI in aseptic RTHA patients compared to the published literature.
\end{abstract}

Project Methods: 169 consecutive aseptic RTHAs performed with modern perioperative and infection-prevention protocols by a single surgeon at a single center were retrospectively reviewed. $80 \%$ of patients were discharged on 7-day oral antibiotic prophylaxis while intra-operative cultures were incubating. Infections and reoperations were documented.

Results: Average age and BMI were 63 years and $30 \mathrm{~kg} / \mathrm{m}^{2} .67 \%$ percent of patients were ASA-III/IV, signifying the severity of comorbidities in this revision cohort. There we no cases of $\mathrm{PJI}$ in the 90-day postoperative period. Ninety-eight percent of cases were infection free at mean follow-up of 45 months. Three (1.8\%) cases underwent reoperation for deep infection at 110 , 161 and 581 days.

Conclusion and Potential Impact: Our observed infection rate of $0.0 \%$ is lower than published infection rates following RTHA and a 1.5\% infection rate in primary THA in patients with no identifiable risk factors for PJI. Based on this clinically meaningful decrease in PJI in this challenging cohort, we encourage further study regarding extended antibiotic protocol weighed appropriately against potential consequences. 